\title{
Exosomal MicroRNA-151a-3p Improves the Sensitivity to Radiotherapy via the Interaction between p53 and Histone Deacetylase 5
}

Seung Min Lee

Asan Medical Center

Bo Hyun yoon

Asan Medical Center

Myoung-Hee Kang

Asan Medical Center

Dong Ha Kim

Asan Medical Center

Yong-Hee cho

Asan Medical Center

Min choi

Asan Medical Center

Jin Woo Lee

Asan Medical Center

Tae Keun Kim

Asan Medical Center

Je-Won ryu

Asan Medical Center

Kyunggon Kim

Asan Medical Center

Young Hoon Sung

Asan Medical Center

Chan-Gi Pack

Asan Medical Center

Jin Kyung rho

Asan Medical Center

Sang-wook Lee

Asan Medical Center

Chang Hoon Ha ( $\square$ chhoonha@amc.seoul.kr)

Asan Medical Center https://orcid.org/0000-0001-7606-4340 
Research

Keywords: Exosome, microRNA, HDAC5, p53, radiotherapy, hepatocellular carcinoma

Posted Date: August 31st, 2021

DOl: https://doi.org/10.21203/rs.3.rs-847360/v1

License: (c) (i) This work is licensed under a Creative Commons Attribution 4.0 International License. Read Full License 


\section{Abstract}

Background: Tumor-derived exosomal microRNAs are key elements of the cell-cell communications response to lots of stimuli. However, various functions of the exosome in tumor suppression by radiotherapy (RT) are not clearly understood. Our study showed a previously unknown interaction of p53 and histone deacetylase 5 (HDAC5) by radiation exposure in hepatocellular carcinoma (HCC).

Methods: Using serial ultracentrifugation methods, radiation and non-radiation exosomes were purified to investigate the radioresistance of miRNA151a-3p. Radiation doses were treated in $2 \mathrm{gy}$ and $4 \mathrm{gy}$ using radiation equipment X-RAD 320 to observe the expression of HDAC5 and p53 in hepatic cancer cells. Exosomal miRNA bioinformatics analysis was conducted to find a variation in the miRNA configuration inside Exosome after radiation exposure.

Results: HDAC5 and p53 interacted by exposure to radiation, which increased exosome release and altered microRNAs' composition within exosomes. Also, we have described the intercommunication occurring between irradiated and untreated cells via exosomal microRNAs that affect tumor proliferation. In particular, the expression of exosomal microR151a-3p was markedly reduced by radiation treatment. We confirmed that inhibition of exosomal microR151a-3p promotes suppression of non-irradiated cancer cells, thereby increasing RT sensitivity.

Conclusion: our present findings demonstrated HDAC5 is a key component of the p53-mediated release of exosomes resulting in tumor suppression through exosomal microRNA-151a-3p in response to radiation. Finally, we highlight the important role of exosomal microRNA 151a-3p as a biomarker in enhancing RT sensitivity.

\section{Background}

The Exosomes are extracellular vesicles of 30-150 nm in size that are released outside of the cell, which structurally constitutes a phospholipid bilayer membrane, so there is no possibility of being broken down by various enzymes in the body. Cancer cells secrete 10 times more exosomes than normal cells, and include growth factors, chemokines, miRNAs, etc. inside exosomes to regulate the microenvironment around cancer, and promote the growth and communication of cancer cells [1, 2]. Recent studies on miRNA contained within exosome have been actively conducted [3,4]. After release, tumor-derived exosomal microRNAs (miRNAs) regulate such functions as retaining tumor growth, invasion, metastasis and therapyresistance in tumor cells through cell-cell communication within the tumor derived exosome. Therefore, exosomal miRNAs have an essential function to mediate tumor progression and can likely be applied as a promising biomarker in the diagnosis and prediction of cancer [5] [6].

p53 is a tumor suppressor that is very important in cancer and regulates cell-cycle control, aging, apoptosis, and genomic stability in response to various external stresses [7, 8]. Recent studies have shown that p53 modification is temporarily regulated by histone deacetylase 5 (HDAC5) [9]. It is believed that various posttranslational modifications of p53 play an important role in determining p53 promoter specificity toward its various target gene sets. During post-translational modifications, Acetylation of p53 at lysine 120 of the 
DNA binding domain has been associated with p53-mediated apoptosis [10]. Further, it is known that the secretion of exosomes in cells undergoing p53 response by stress is increased by effector TSAP6 downstream of $\mathrm{p} 53$. So, exosomes can be regulated by the p53 response $[11,12]$.

Histone acetylation/deacetylation plays a critical role in the regulation of gene expression [13] [14]. In particular, HDAC5, one of class II HDACs, has been implicated in modulating cellular signalings by interactions with various transcriptional cofactors and confers responsiveness to gene expressions. Previous studies have shown that tumor suppressor p53 is activated by DNA damage that causes genotoxicity, and as a temporary regulator, HDAC5 interacts with p53 and HDAC5 in the early stages of stress, thereby deacetylating $\mathrm{p} 53$. The pro-apoptotic target gene, modulated by $\mathrm{p} 53$, is transactivated by k120 deacetylation of p53 by HDAC5, which determines the fate of the cell as a regulator of p53 modification $[9,15]$. However, little is known about the role of HDAC5 in response to radiation therapy in tumor.

Radiotherapy (RT) is one of the most commonly used therapeutic methods that can suppress the proliferation of cancer cells. However, there may be some side effects that reduce sensitivity to RT and become radiation resistant. In recent years, the goal of RT is to improve the quality of life of patients by reducing the side effects of radiation treatment. In particular, in hepatocellular carcinoma (HCC), RT has become a key treatment that prevents the progression of cancer and helps surgical treatment. RT is the most effective treatment for local tumor metastasis in patients who potentially need liver resection or transplantation $[16,17]$.

Here, we showed our findings regarding tumor-derived exosomes, including the miRNA delivering mechanism. We then described the interactions that occur between irradiated and untreated cells via exosomal miRNAs that affect tumor proliferation. HDAC5 and p53 interacted by exposure to radiation, the effect of which increased exosome release and altered the composition of miRNAs within exosomes. In particular, the expression of Exosomal miRNA 151a-3p was decreased, which is the result of irradiation. As a result of the experiment, it was confirmed that inhibition of exosomal miRNA 151a-3p promotes apoptosis of non-irradiated tumor cells, thereby enhancing the RT effect. Finally, we highlight the potential role of miRNA-151a-3p as biomarkers in tumor therapeutics and sensitivity to RT.

\section{Methods}

\section{Cell culture, X-Radiation treatment}

HepG2, SK-Hep1 was purchased from the American Type Culture Collection (Rockville, MD) (ATCC HTB177). Hep3B was purchased by Korea Cellular Bank (seoul, Korea). It was cultured in DMEM (Corning) with $10 \%$ fetal bovine serum, $4.5 \mathrm{~g} / \mathrm{L}$ glucose, L-glutamine, sodium pyruvate, and $100 \mathrm{U} / \mathrm{mL}$ of penicillin and streptomycin, and maintained at $37^{\circ} \mathrm{C}$ in a humidified chamber containing $5 \% \mathrm{CO} 2$. Radiation was exposed with 2Gray, 4Gray a using X-RAD320 (1Gray/min at 320KV, 12.5mA, 50cm SSD (HVL $\approx 4 \mathrm{~mm} \mathrm{Cu})$ ) equipment. 


\section{Exosome purification}

Radiation and non-radiation exosomes purified using ultracentrifugation methods. $1 \times 106$ cells were seeded in $100 \mathrm{~mm}$ dishes and allowed to recover for overnight. Then, the cells were washed twice with prewarmed PBS, and the culture medium was replaced with exosome-free medium supplemented with $5 \%$ exosome-depleted FBS (SBI). The Radiation exosome is treated 4Gray Radiation using X-RAD 320 ( $1 \mathrm{Gray} / \mathrm{min}$ at $320 \mathrm{KV}, 12.5 \mathrm{~mA}, 50 \mathrm{~cm} \mathrm{SSD}(\mathrm{HVL} \approx 4 \mathrm{~mm} \mathrm{Cu})$ ) equipment. The medium was gathered and

subjected to gradient centrifugation. Briefly, the medium was first centrifuged at $1000 \mathrm{~g}$ for $10 \mathrm{~min}$, at $3000 \mathrm{~g}$ for 30 min, then centrifuged in a Beckman Coulter Optima ${ }^{\mathrm{TM}}$ L-XP Ultracentrifuge System at 100,000 gavg at $4^{\circ} \mathrm{C}$ for 90 minutes with a SW 28 Swinging-Bucket Rotor (k-factor: 71) to pellet exosomes. The supernatant was carefully removed, and crude exosome-containing pellets were resuspended in $1 \mathrm{~mL}$ of ice-cold PBS and pooled. A second round of ultracentrifugation $\left[100,000\right.$ gavg at $4^{\circ} \mathrm{C}$ for 90 minutes with a Type SW 28 Swinging-Bucket Rotor ( $k$-factor: 71)] was carried out, and the resulting exosome pellet resuspended in 500 $\mu$ l of PBS.

\section{Western blot analysis}

The cells were collected, washed with PBS, and lysed in lysis buffer containing protease inhibitors (GenDEPOT, USA). After determining the protein concentration with a Bradford Protein Assay Reagent (BioRad), equal amounts of protein were separated on $10 \%$ SDS-PAGE, electrically transferred to nitrocellulose membrane, and blocked with $5 \%$ skim milk and $5 \%$ BSA. The membranes were incubated with anti-HDAC5 (1:1000; Cell Signaling Technology, USA), anti-p53 (1:2000; Santacruz, USA), anti-p21 (1:2000; Cell Signaling Technology), anti-Puma (1:2000; Cell Signaling Technology), anti-STEAP3 (1:1000; Proteintech, USA), anti-Maspin (1:1000; Cell Signaling Technology), anti-CD63 (1:1000; Santacruz), anti- CD81 (1:1000; Santacruz), anti-HSP90 (1:1000; BD bioscience), anti-BCL2 (1:1000; Cell Signaling Technology), anti-Ecadherin (1:1000; Agilent Dako), anti-Twist1 (1:1000; abcam), N-cadherin (1:1000; Cell Signaling Technology), Vimentin (1:1000; Calbiochem) or anti-beta-actin (1:1000; Santacruz) primary antibody overnight shaking at $4{ }^{\circ} \mathrm{C}$. After washing twice, the membranes were then incubated with horseradish peroxidase (HRP)-conjugated anti-rabbit and anti-mouse secondary antibody (Jackson ImmunoResearch Inc. USA) at room temperature for 1 hour. Finally, the membranes were incubated with WESTSAVE-UP western blotting substrate (Youngin frontier, Korea), and images were visualized using ChemiDoc imaging system (Bio-Rad, USA) and recorded.

\section{Duolink proximity ligation assay (PLA)}

The DuoLink® In Situ Red Starter Kit Mouse/Rabbit (DU092101, Sigma-Aldrich, Darmstadt, Germany) was used to detect interacting target proteins. Cells were seeded in eight-well chamber removable slides (ibidi GmbH Am Klopferspitz, Germany) and cultured 24hours. Then, HepG2 cells were exposed to $4 \mathrm{~g}$ of radiation, and after 12 hours, the slides were washed with cold 1xPBS and fixed in $4 \%$ paraformaldehyde for 30 minutes. Then slides were blocked with Duolink Blocking Solution in a pre-heated humidified 
chamber for 30 min at $37^{\circ} \mathrm{C}$. The primary antibody to detect HDAC5 and p53 was added to the slides and incubated overnight at $4^{\circ} \mathrm{C}$. Then slides were washed with $1 \times$ Wash Buffer $A$ and subsequently incubated with the two PLA probes (1:5 diluted in antibody diluents) for $1 \mathrm{~h}$, then the Ligation-Ligase solution for 30 min, and the Amplification-Polymerase solution for $100 \mathrm{~min}$ in a pre-heated humidified chamber at $37^{\circ} \mathrm{C}$. Before imaging, slides were washed with $1 \times$ Wash Buffer $B$ and mounted with a cover slip using Duolink In Situ Mounting Medium with DAPI. Fluorescence images were acquired using a zeiss LSM 780 confocal microscope. 17

\section{Transmission electron microscopy (TEM)}

In order to photograph exosomes, $0.1 \mathrm{M}$ of exosome pellets were fixed in $2.5 \%$ glutaraldehyde in $0.1 \mathrm{M}$ cacodylate solution ( $\mathrm{pH} \mathrm{7.0)}$ ) for 1 hour and then fixed in $2 \%$ osmium tetroxide for another hour. $\left(4^{\circ} \mathrm{C}\right) \mathrm{After}$ dehydration using the graded acetone series, embedding was performed through Spurr's medium (Electron Microscopy Sciences). The resulting section sample was cut at $60 \mathrm{~nm}$ with an ultramicrotome (RMC MTXL, USA), followed by double staining using $2 \%$ uranyl acetate for 20 minutes and citrate for 10 minutes. Prepared sections were photographed at 80 kV using Hitachi H-7600 TEM (Hitachi, Japan) equipment.

\section{Exosome size distribution and concentration measurement.}

To determine exosome size distribution and concentration, nanoparticle tracking-based analyses were performed using a NanoSight (NS500) apparatus (Malvern Instruments Ltd.). Samples were diluted to provide counts within the linear range of the instrument. The videos of 1-minute duration were recorded for each sample, with a frame rate of 30 frames per second. Particle movement was analyzed by NTA software (NTA 2.3; NanoSight Ltd.) according to the manufacturer's protocol. The NTA software was optimized to first identify and then track each particle on a frame-by-frame basis. 18, 19

\section{Small RNA sequencing}

\section{RNA isolation}

HepG2-derived Exosomal small RNA was extracted using a miRNeasy Mini Kit (Qiagen Korea Ltd.) according to the manufacturer's instructions. RNA quality was assessed by Agilent 2100 bioanalyzer using the RNA 6000 Pico Chip (Agilent Technologies, Amstelveen, The Netherlands), and RNA quantification was performed using a NanoDrop 2000 Spectrophotometer system (Thermo Fisher Scientific, Waltham, MA, USA).

\section{Library preparation and sequencing}


For control and radiation induced HepG2-drived exosomal small RNAs, the construction of the library was performed using NEBNext Multiplex Small RNA Library Prep kit (New England BioLabs, Inc., USA) according to the manufacturer's instructions. Briefly, for library construction, total RNA from each samples were used $1 \mathrm{ug}$ to ligate the adaptors and then CDNA was synthesized using reverse-transcriptase with adaptor-specific primers. PCR was performed for library amplification and libraries were carried out clean-up using QIAquick PCR Purification Kit (Quaigen, Inc, German) and AMPure XP beads (Beckmancoulter, Inc., USA). The yield and size distribution of the small RNA libraries were assessed by the Agilent 2100 Bioanalyzer instrument for the High-sensitivity DNA Assay (Agilent Technologies, Inc., USA). High-throughput sequences were produced by NextSeq500 system as way of single-end 75 sequencing (Illumina, SanDiego, CA., USA).

\section{Data analysis}

Sequence reads were mapped by bowtie2 software tool in order to obtain bam file. Mature miRNA sequence is used as a reference for mapping. Read counts mapped on mature miRNA sequence were extracted from the alignment file using bedtools v2.25.0 (Quinlan AR, 2010) and Bioconductor 20 that uses R statistical programming language ( $R$ development Core Team, 2016). Read counts were used in order to determine the expression level of miRNAs. Quantile normalization method was used for comparison between samples. For miRNA target study, miRWalk 2.021 was performed. Functional gene classification was performed by DIANA 22.

Exosomal small RNA sequencing arrays were prepared, hybridized, and scanned at the local authorized Illumina array service provider (Ebiogen, Seoul, South Korea).

\section{TCGA(The Cancer Genome Atlas) and GEO(Gene Expression Omnibus) Database analysis}

The two gene expression profiling data sets (GSE74618, GSE147889) we analyzed were downloaded from the NCBI GEO database.23 The GSE74618 database consists of 218 human HCC tumours samples, 10 adjacent cirrhotic non-tumoral tissue samples, and 10 healthy liver samples. We used adjacent cirrhotic non-tumoral tissue samples and healthy liver samples as controls and compared them with human HCC tumours samples. The GSE1477889 database consists of 97 samples of HCC tumours and 97 samples of the same patient's surrounding chronic hepatitis tissue. Surrounding chronic hepatitis tissue was used as a control and compared with HCC tumour samples. Two data sets were analyzed as volcano plots and mean difference plots, respectively, using the GEO2R analysis tool. TCGA (The Cancer Genome Atlas) data analysis was performed using oncomiR (WashU Pan-Cancer miRNome Atlas) online bioinformatics tool.24 Among the TCGA data, the analysis was conducted using the LIHC (Liver Hepatocellular Carcinoma) database $(n=366)$, and the days' survival rate graph of liver cancer patients was exhibited. 


\section{Kyoto encyclopedia of genes and genomes (KEGG) pathway enrichment analyses}

KEGG is a database that contains genetic information on the human genome, biological cell signalling pathways, and various diseases and chemicals. The KEGG pathway analysis of exosomal miRNA was analyzed using the DIANA-miRPath v3.0 analysis tool. DIANA-miRPath v3.0 is a KEGG-based miRNA pathway prediction tool that actively interacts with the major miRNA analysis tools, DIANA-microT CDS and TargetScan v6.2 database, to analyze the target pathway.22

\section{miRNA Transfection}

Cells were seeded in 6 -well plates at $1 \times 105 \mathrm{cells} / \mathrm{mL} /$ well before the transfection and radiation. On the following day, After HepG2 cells were exposed 4gy of radiation using X-RAD 320 and transfection was performed when the cells had reached approximately 80\% confluence. miRCURY LNA miR-151a-3p mimic (miRCURY LNA miRNA Mimic, MIMAT0000757) were purchased from QiAGEN (Qiagen Korea Ltd.). And scrambled miRNA control were purchased from BIONEER (Seoul, Korea). The final concentrations of miRNA were 50-100nM Transfections were conducted with Lipofectamine 3000 (Invitrogen) according to the manufacturer's instructions.

\section{Quantitative real time reverse transcription polymerase chain reaction(qRT-PCR)}

Total RNA was extracted using a miRNeasy Mini Kit (Qiagen Korea Ltd.) in the manufacturers' protocols. For the quantification of matured cellular and exosomal miRNAs, the extracted total RNA was polyadenylated with a poly(A) tailing kit (Ambion, Austin, TX) prior to reverse transcription. And was reverse transcribed to cDNA using PrimeScript ${ }^{\text {TM }}$ Reverse Transcriptase (Takara Bio Inc.). The cDNA samples were used for quantitative RT-PCR analysis in triplicate to determine the expression levels of miR-151a-3p using a TB Green ${ }^{\circledR}$ Premix Ex Taq ${ }^{\text {TM }}$ (Takara Bio Inc.) and CFX96 Detection System real-time PCR instrument.

\section{Proliferation assay}

The cell proliferation assay was performed using the Chromo-CK Cell Viability Assay KIT (monobio seoul, korea) according to the manufacturer's instructions. Transfected cells were harvested at the designated times after seeding. Briefly, the reagent $(10 \mu \mathrm{l} /$ well) was added to $100 \mu \mathrm{l}$ of medium containing cells in each well of a 96-well plate and incubated for 1 hours at $37^{\circ} \mathrm{C}$ under humidified $5 \% \mathrm{CO} 2$ in air. For colorimetric analysis, absorbance at $450 \mathrm{~nm}$ was recorded using an ELx800 Absorbance Microplate Reader (SpectraMax 340PC Microplate Reader, Molecular Devices, USA). Each experiment was repeated at least 3 times. 


\section{Migration invasion assay}

Tumor cell migration and invasion were analyzed in 24-well plates with 8- $\mu \mathrm{m}$ pore size polycarbonate membranes (BD, NJ, USA). For invasion assays, the membranes were coated with diluted Matrigel (BD, NJ, USA) to form matrix barriers. HepG2 were transfected with 151a-3p or negative control. For the migration and invasion assays, the cells $\left(5 \times 10^{\wedge} 4\right.$ for migration, $1 \times 10^{\wedge} 5$ for invasion) were resuspended in $200 \mu \mathrm{l}$ of serum-free DMEM at 24 hours post-transfection and added to the upper compartments of the chambers, and the lower compartments were filled with $600 \mu$ l of DMEM with $10 \%$ FBS (different substrates were added accordingly). After incubation at $37^{\circ} \mathrm{C}$ for 10 hours (migration) or 24 hours (invasion), the cells remaining on the upper surfaces of the membrane were removed. The cells on the lower surfaces of the membrane were fixed, stained with crystal violet and counted under a light microscope. Each experiment was repeated at least 3 times.

\section{Analysis of cell cycle}

The Transfected cells were collected and resuspended after radiation exposure. Annexin V-fluorescein isothiocyanate (FITC) and propidium iodide (PI) staining assays were conducted to detect the percentage of apoptotic (FITC-stained) and necrotic (Pl-stained) cells in a given population. Analyses were performed by a FACS canto flow cytometer (BD Biosciences, Franklin Lakes, NJ, USA) according to the manufacturer's instructions. The procedures were repeated in triplicate. 25

\section{Analysis of cell apoptosis}

The Transfected cells were collected and resuspended with cold PBS after radiation exposure. And fixed in cold $70 \%$ ethanol added dropwise to the cell pellet while vortexing. Stored at $-20^{\circ} \mathrm{C}$ for overnight. Cells were rehydrated with PBS for 10 min at RT and then cells were stained with propidium iodide (PI) staining solution contained with $50 \mu \mathrm{g} / \mathrm{ml} \mathrm{PI} \mathrm{(BD} \mathrm{Biosciences,} \mathrm{Franklin} \mathrm{Lakes,} \mathrm{NJ,} \mathrm{USA),} 100 \mu \mathrm{g} / \mathrm{ml}$ RNase A, DNase and protease-free (Thermo Scientific) in 1X binding buffer. Analyses were performed by a FACS canto flow cytometer (BD Biosciences, Franklin Lakes, NJ, USA) according to the manufacturer's instructions. The procedures were repeated in triplicate.

\section{Subcutaneous tumor xenograft models}

BALB/c nude mice (5-week old male) were purchased from the Charles River Laboratories, Inc (Wilmington, Massachusetts). The fodder was feed to the normal rodent laboratory animal feed. $5 \times 10^{\wedge} 6$ HepG 2 cells in total volume $100 \mathrm{ul}$ were injected into the right flank of a 5 -week-old nude mouse in a 1:1 ratio with Matrigel (corning 356234). After the average of the transplanted solid cancer size increased by more than $100 \mathrm{~mm}$, the invivo-jetPEl/target miRNA complex was treated. invivo-jetPEl was used as an efficient carrier for delivering miRNA and was mixed with $5 \%$ glucose solution at a ratio of miRNA 10ug / invivo-jetPEl 1.2ul and treated in a total dose of $100 \mathrm{ul}$. The invivo-jetPEI/target miRNA complex was administered 3 times 
every 3 days by intratumoral injection. Tumor size was measured using a standard ABS digimatic caliper (CD-15AX) every 3 days after transplantation. Tumor volume was measured using the following formula: $\Pi$ $x 4 / 3 x$ larger diameter $x$ smaller diameter square. At the time when the volume of the largest tumor of the implanted nude mice in the experimental group exceeded $1000 \mathrm{~mm} 3$, the tumor was separated after sacrifice, the weight of the tumor was measured, and expression of the target protein in cancer tissue was analyzed.

\section{Statistical analysis}

Each experiment in our study was repeated three times. Mathematical data are expressed as mean \pm SD. Unless indicated, the differences between two groups were analyzed using a Student's t-test (two-tailed). Unless otherwise noted, significant numerical differences between the two groups were analyzed using Student's t-test (two-tailed). The overall survival curve was graphed using the Kaplan-Meier method and compared through the log-rank test. Differences were evaluated statistically significant at $P<0.05$. All statistical analysis was performed using SPSS13.0 software (SPSS, Chicago, IL, USA).

\section{Results}

\section{Modulation of p53 and HDAC5 by RT}

An experiment was conducted to verify that protein p53 and HDAC5 were modulated by radiation in HCC. Radiation doses were treated in 2gy and 4gy using radiation equipment X-RAD 320 to observe the expression of HDAC5 and p53 when radiation was treated in three types of hepatic cancer cells. The interaction of HDAC5 and p53 by DNA damage has been previously described [9]. However, the interaction of HDAC5 and p53 in HCC by radiation is not yet known. HepG2 and SK-Hep1 are p53 wild types (p53+/+) and Hep3B are $\mathrm{p} 53$ deletion types (p53-/-). The amount of expression of each protein was measured through the Western Blot in order of 6 hours, 12 hours, 18 hours, and 24 hours (Fig. 1A-F). In HepG2, the expressions of HDAC5 and p53 were significantly increased from 6 hours compared to the control group by radiation 2gy and 4gy. Later, at 2 gy of radiation, HDAC5 showed a time-dependent decrease again. However, HDAC5 and p53 were significantly increased in radiation 4gy regardless of time. This shows that the expression of HDAC5 and p53 and its downstream is regulated by irradiation in HepG2 (Fig. 1. A,B). SKHep1 increased the expression of HDAC5 24 hours after 2gy irradiation. However, in $4 \mathrm{gy}$, it was reduced compared to the control group. p53 and its downstream showed a significant increase by irradiation, but HDAC5 did not show a significant expression pattern (Fig. 1. C, D). Finally, Hep3B showed an independent increase in HDAC5 expression without p53 after irradiation. As a result, it was confirmed that among p53 and HDAC5 increased by irradiation, HDAC5 independently increased protein expression (Fig. 1E, F).

Subsequent experiments were conducted on HepG2, which showed the most significant change by RT among the three liver cancer cells. To check the relationship between HDAC5 and p53, a HepG2 cell line that p53-/- was created using the CRISPER CAS9 system. The target site of Human p53:

TGTAACAGTTCCTGCATGGG was investigated by the NGS method. The insertion/deletion frequency was

Page 10/30 
99.98\% (T insertion:19.9\%, CCTG deletion:18.8\%, AT insertion:19.5\%), and the elimination of the p53 gene was confirmed to be close to perfection (supplementary Fig. 1). The radiation was exposed for $\mathrm{p} 53+/+$, p53-/-cells and measured the protein expression of HDAC5 and p53, p21, Puma with western blot. The expression of p53, p21 did not almost appear because p53 was deleted. Regardless of p53 identified an increase in the expression of HDAC5. As a result, it was confirmed that the expression of HDAC5 was increased by irradiation regardless of the expression of p53 in cells, which showed the most significant expression by irradiation, and the relationship between HDAC5 and p53 was identified (Fig. 1. G, H). Using FACS equipment, it was confirmed that cell cycle arrest and apoptosis are induced in p53+/+, p53-/-cells by radiation treatment. In p53+/+cell, powerful cell cycle arrest in the G2/M phase was observed from 6 hours after irradiation and gradually switched to $\mathrm{G} 1$ phase cell cycle arrest as time passed. A similar cell cycle alteration was observed in p53-/-cell, but it was verified that the effect was smaller than that of $\mathrm{p} 53+/+$ cell. Also, p53-/-cell had significantly less apoptosis 48 hours after irradiation than p53+/+cell. It showed stronger cell cycle arrest and apoptosis at p53+/+cell compared to p53-/-cell (supplementary Fig. 2).

\section{Intercommunication between p53 and HDAC5 by RT resulting in increasing the expression of TSAP6, exosome modulating protein}

To identify the more accurate interaction between p53 and HDAC5, this was investigated using the duolink PLA assay. When the cells were not exposed to radiation, no red dots were found to interact, and $4 \mathrm{~Gy}$ of radiation revealed multiple red dots, the interaction between p53 and HDAC5. It has been demonstrated that radiation exposure induces a significant increase in the interaction of p53 and HDAC5 in cells (Fig. 2. A). Using western blot validated whether TSAP6 (Tumor Suppressor Activation Pathway 6), an exosomemodulated protein in the direct downstream of p53 and controlled by p53 is increased by RT $[11,12]$. The expression of TSAP6 was verified with a western blot at 12 hours and 24 hours after exposure to 4Gy of radiation. Our results showed that the interaction between HDAC5 and p53 was enhanced by radiation, and the downstream TSAP6 of p53 was also significantly increased (Fig. 2. B, C).

The characteristics of exosomes derived from p53+/+, p53-/-cells were identified. Exosomes secreted by cells were observed using an electron microscope. (Fig. 2. D) Western blot analysis showed that microvesicles released from p53+/+, p53-/-cells expressed exosome markers CD63, CD81, and HSP90. All exosome markers have been demonstrated [18]. We also identified the discriminating expression of protein maspin (mammary serine protease inhibitor), which is released by p53 dependently through exosome by radiation exposure [11]. Inside p53+/+cell derived exosomes, it was found that the expression of maspin was increased by radiation. However, p53-/-cell derived exosomes demonstrated no expression of maspin by radiation exposure. (Fig. 2. E, F) The expression of maspin inside the exosome induced by radiation exposure is regulated by the expression of p53. p53 directly binds to the p53 gene-consensus binding site present in the maspin promoter, through activating the maspin promoter and inducing maspin expression [19].

\section{Exosome secretion in a p53-dependent manner by RT}


After exposure to radiation, experiments were conducted to demonstrate that the secretion of exosome changes over time. The number of cultured p53+/+cells and p53-/-cells was equal to $5 \times 10^{6}$. Experiments were conducted at $6,12,24$, and 48 hour intervals after exposure to $4 \mathrm{~Gy}$ of radiation, and exosomes released at each hour were extracted by ultracentrifugation. The qualitative and quantitative comparison and analysis of the isolated exosomes were performed using the Nanosight NT300 equipment (supplementary Fig. 3. A, B). As a result of the analysis, the Exosome showed a heterogeneous exosomal population with a diameter of 30-300 nm or more. These results suggest that the isolated exosomes had high purity and satisfy the size, density, structure, and characteristics of vesicles secreted by cells [18]. p53+/+cells manifested a significant increase in the secretion of overall vesicles when exposed to radiation of $4 \mathrm{~Gy}$. As a result of comparing the total vesicles by each secreted time, it was found that the volume of secreted vesicles increased more than twice when exposed to radiation. In particular, 24 hours after exposure to radiation, the total quantity of vesicle secretion was found to be more than three-fold that of the control, and the most vesicle secretion exhibited at this time (Fig. 3A). Conversely, p53-/-cells exhibited a decrease in the volume of vesicle secretion after exposure to radiation. From 6 hours to 48 hours, the quantity of vesicle released in all experimental groups was significantly decreased compared to the control (Fig. 3B). The secreted extracellular vesicles were analyzed qualitatively by separating them into $30-150 \mathrm{~nm}$, 150-300 $\mathrm{nm}$, and $>300 \mathrm{~nm}$ by size. The p53+/+cells secreted Exosome size part (30-150nm) was the most significant increase of 12 hours after radiation exposure. As time passed after exposure to radiation, the variation of vesicles was characterized by a change in the direction of increasing their size. It was verified that exosome-sized vesicles were noticeably reduced in vesicles 48 hours after exposure to radiation (Fig. 3C). In the same way, p53-/-cells derived exosome was analyzed by dividing each size. The exosome-sized vesicles released after irradiation were also rapidly reduced (Fig. 3D). The exosomes of p53+/+ and p53-/cells released after irradiation were analyzed qualitatively and quantitatively. (Fig. 3E, F) This experiment proved that the secretion of extracellular vesicles was increased by radiation exposure. Among them, 30$150 \mathrm{~nm}$-sized exosomes proved to be increased or decreased by p53 dependency. This result suggests that the expression of p53 induced by RT increases exosome secretion through the TSAP6 pathway.

\section{Alteration of exosomal miRNA's expressions by RT in HCC}

After the release in a cell, exosomes are taken up by neighboring or distant cells, and the miRNAs contained within modulating such processes as interfering with tumor immunity and the microenvironment, possibly facilitating tumor growth, invasion, metastasis, angiogenesis, and drug resistance. Therefore, exosomal miRNAs have a significant function in regulating tumor progression. Exosomal miRNA bioinformatics analysis was conducted to find a variation in the miRNA configuration inside Exosome after radiation exposure. The variation of miRNA in exosome secreted from the radiation exposed cell and miRNA in exosome secreted from without radiation exposure was analyzed through Heatmap utilizing MeV software (Small RNA sequencing, miRNA-Seq Only Analysis Program developed by Dana-Farber Cancer Institute in the United States) (supplementary Fig. 4. A). Also, to find out which intracellular function each of the miRNAs inside the exosome changed after irradiation, it was classified and analyzed into various cell functional categories using Gene Ontology terms (supplementary Fig. 4. B). Handling the miRNA's fold change values analyzed, the graph was composed in descending order (Fig. 4. A). It has been confirmed that 33 miRNAs have significantly reduced due to irradiation among 156 miRNAs that have inside exosome

Page 12/30 
(Fig. 4. B). Among the miRNAs with reduced expression, we analyzed using the hepatocellular carcinoma GEO data set to identify the miRNAs associated with the progression of hepatocellular carcinoma. The GEO data set was downloaded from NCBI Gene Expression Omnibus, and analyzed by volcano plot and meandifference plot using the GEO2R analysis tool (supplementary Fig. 5. A, B). As a result of analysis utilizing the HCC miRNA GEO data set (Spain, GSE74618), it was verified that 4 specific miRNAs (miR-151a-3p, miR106b-5p, miR-183-5p, miR-452-5p) were significantly increased in tumor tissues compared to normal (Fig. 4.C) [20]. The HCC miRNA GEO data set (Spain, GSE74618) was re-validated using another HCC miRNA GEO data set (Japan, GSE147889) to confirm the accuracy of the analysis results (fig S4.B). To ascertain the relationship between the four miRNAs (miRNA151a-3p, miRNA 106b-5p, miRNA183-5p, miRNA 452-5p) with increased expression in $\mathrm{HCC}$ and the survival rate of liver cancer patients, we analyzed using OncomiR Cancer miRNome Atlas. As a result of OncomiR analysis, it was found that the higher the expression of miRNA151a-3p, miRNA 106b-5p, miRNA183-5p, and miRNA 452-5p in the tumour tissues of liver cancer patients, the significantly decreased the survival rate of liver cancer patients (Fig. 4.D) [21]. We performed the KEGG pathway analysis to find out which molecular biological processes the four miRNAs, which were directly related to the prognosis of liver cancer patients, affect cancer (Table 1). Among the analysis results performed, the cell cycle, a mechanism directly related to radiation therapy, and the p53 signalling pathway, the central target of our study, was ascertained to be significant signaling mechanisms. In addition, targetscan (http://www.targetscan.org/vert_72/) analysis tool was used to analyze the target protein of each miRNA. As a result of the target analysis of miRNA, it was discovered that miRNA151a-3p can bind to the p53 mRNA target region with a high probability (Fig. 4E). Likewise, in previous studies, it has been reported that miRNA 151a-3p was bioinformatically analyzed as a target miRNA of p53 and binds to the p53 mRNA target region, thereby inhibiting the expression of p53 in cells $[22,23]$. We considered miRNA $151 a-3 p$ interesting and continued the study, and it was confirmed that miRNA $151 a-3 p$ is deeply related to tumorigenesis of various cancers (Table. 2). Furthermore, three cancer types were identified, where patient survival was significantly associated with miRNA 151a-3p (Table. 3). As expected, miRNA 151a-3p had a great correlation with the prognosis of liver cancer patients. So, it has been confirmed that miRNA 151a-3p is a respectable relation to tumorigenesis and survival of Liver Hepatocellular carcinoma (LIHC). We actually verified that miRNA 151a-3p was reduced by radiation exposure both intracellularly and in exosomes. The expression of miRNA 151a-3p in cell and exosome after radiation exposure was analyzed using qRT-PCR. The quantification of miRNA 151a-3p was quantified using miR-Let7a [24]. Cellular miRNA 151a-3p decreased by $1 / 2$ after radiation exposure, whereas exosomal miRNA 151a-3p decreased by more than 1/4 after radiation exposure. (Fig. 4. F, G) As a result, miRNA 151a-3p had a significant relationship with the poor survival of HCC. It also after irradiation in hepG2, a HCC cell line, demonstrated a significant decrease in the expression of miRNA 151a-3p inside the cellular and exosome.

\section{Table 1}

\section{Results of KEGG pathway analysis of major miRNAs within exosomes that were reduced after radiation exposure.}




\begin{tabular}{|c|c|c|}
\hline KEGG pathway & p-value & Genes \\
\hline Pathways in cancer & $6.61 \mathrm{E}-08$ & 103 \\
\hline Viral carcinogenesis & $1.62 \mathrm{E}-06$ & 56 \\
\hline Cell cycle & $1.88 \mathrm{E}-06$ & 43 \\
\hline Adherens junction & $1.88 \mathrm{E}-06$ & 26 \\
\hline Colorectal cancer & 2.49E-06 & 25 \\
\hline Hepatitis B & 2.01E-05 & 45 \\
\hline Thyroid cancer & 2.61E-05 & 15 \\
\hline TGF-beta signaling pathway & 0.000154 & 27 \\
\hline Bladder cancer & 0.000154 & 19 \\
\hline p53 signaling pathway & 0.000226 & 27 \\
\hline Wnt signaling pathway & 0.000238 & 43 \\
\hline Endometrial cancer & 0.000245 & 19 \\
\hline Renal cell carcinoma & 0.000284 & 24 \\
\hline PI3K-Akt signaling pathway & 0.000379 & 87 \\
\hline RNA transport & 0.000401 & 48 \\
\hline Protein processing in endoplasmic reticulum & 0.000401 & 50 \\
\hline AMPK signaling pathway & 0.000401 & 40 \\
\hline Pancreatic cancer & 0.001437 & 24 \\
\hline Insulin signaling pathway & 0.002105 & 41 \\
\hline mTOR signaling pathway & 0.002607 & 22 \\
\hline Focal adhesion & 0.002607 & 55 \\
\hline Small cell lung cancer & 0.002778 & 27 \\
\hline Non-small cell lung cancer & 0.006262 & 18 \\
\hline Endocytosis & 0.006754 & 50 \\
\hline Sphingolipid signaling pathway & 0.008416 & 32 \\
\hline mRNA surveillance pathway & 0.014393 & 27 \\
\hline Hepatitis C & 0.015025 & 37 \\
\hline Phosphatidylinositol signaling system & 0.024036 & 20 \\
\hline MAPK signaling pathway & 0.024051 & 59 \\
\hline
\end{tabular}

Page 14/30 
Table 2

15 cancer types where tumorigenesis is significantly associated with hsa-miR-151a-3p expression.

Table 3

cancer types where survival is significantly associated with hsa-miR-151a-3p.

\begin{tabular}{|c|c|c|c|c|c|c|c|c|}
\hline $\begin{array}{l}\text { Cancer } \\
\text { Abbreviation }\end{array}$ & $\begin{array}{l}\text { Log } \\
\text { Rank } \\
\text { P- } \\
\text { value }\end{array}$ & $\begin{array}{l}\text { Log } \\
\text { Rank } \\
\text { FDR }\end{array}$ & $\begin{array}{l}\text { Z- } \\
\text { score }\end{array}$ & $\begin{array}{l}\text { Upregulated } \\
\text { in: }\end{array}$ & $\begin{array}{l}\text { Deceased } \\
\text { Expression }\end{array}$ & $\begin{array}{l}\text { Living } \\
\text { Expression }\end{array}$ & $\begin{array}{l}\text { T-Test } \\
\mathrm{P}- \\
\text { value }\end{array}$ & $\begin{array}{l}\text { T-Test } \\
\text { FDR }\end{array}$ \\
\hline LGG & $\begin{array}{l}4.71 \mathrm{e}- \\
02\end{array}$ & $\begin{array}{l}3.48 \mathrm{e}- \\
02\end{array}$ & 1.990 & Deceased & 10.37 & 10.15 & $\begin{array}{l}4.53 e- \\
02\end{array}$ & $\begin{array}{l}1.98 \mathrm{e}- \\
01\end{array}$ \\
\hline LIHC & $\begin{array}{l}5.47 \mathrm{e}- \\
04\end{array}$ & $\begin{array}{l}1.71 \mathrm{e}- \\
02\end{array}$ & 3.471 & Deceased & 11.68 & 11.34 & $\begin{array}{l}3.93 e- \\
03\end{array}$ & $\begin{array}{l}7.93 \mathrm{e}- \\
02\end{array}$ \\
\hline SARC & $\begin{array}{l}8.84 \mathrm{e}- \\
04\end{array}$ & $\begin{array}{l}5.62 \mathrm{e}- \\
01\end{array}$ & 3.320 & Deceased & 11.00 & 10.65 & $\begin{array}{l}2.15 \mathrm{e}- \\
02\end{array}$ & $\begin{array}{l}5.56 \mathrm{e}- \\
01\end{array}$ \\
\hline
\end{tabular}

LGG: brain lower grade glioma; LIHC: liver hepatocellular carcinoma; SARC: sarcoma

\section{Radiotherapy reduced expression of exosomal miRNA-151a- $3 p$ resulting in tumor suppression}

We investigated the radioresistance of miRNA151a-3p. A protein related to cell apoptosis and cell cycle arrest that can be caused by radiation was verified 24 hours after the transfection of negative control and miRNA151a-3p in the cell through the western blot. Cancer repression gene p53 significantly reduced. A Decrease of P21 which inhibits cyclin-dependent phosphorylation to stop cell cycle has been identified. The BCL-2 controlling the Mitochondrial Outer Membrane Permeabilization Pore, which creates a channel that increases the permeability of the mitochondrial outer membrane, has been increased. And Puma associated with apoptosis has been reduced (Fig. 5. A, B). Based on the results of Fig. 5. A-B, the cell cycle arrest analysis experiment was conducted using FACS to verify the function of miRNA151a-3p in cell cycle arrest induced by radiation exposure. 24 hours after miRNA151a-3p transfection, 4Gy radiation was exposed to the cell. After six hours, the cell cycle of each experimental group was analyzed (Fig. 5. C). The G2/M phase arrest was increased in the N.C group and the 4Gy positive group. In the miR-151a-3p group, the G2/M phase arrest was reduced relatively (Fig. 5. D). Also, it has been confirmed that miRNA151a-3p has a function to inhibit cell apoptosis induced by radiation exposure. As a result of the experiment, the miRNA151a-3p group significantly reduced total cell apoptosis compared to the N.C. and 4Gy positive groups (Fig. 5. E-F). 


\begin{tabular}{|c|c|c|c|c|c|}
\hline $\begin{array}{l}\text { Cancer } \\
\text { Abbreviation }\end{array}$ & $\begin{array}{l}\text { T-Test } \\
\text { P-value }\end{array}$ & $\begin{array}{l}\text { T-Test } \\
\text { FDR }\end{array}$ & $\begin{array}{l}\text { Upregulated } \\
\text { in: }\end{array}$ & $\begin{array}{l}\text { Tumor } \\
\text { Expression }\end{array}$ & $\begin{array}{l}\text { Normal } \\
\text { Expression }\end{array}$ \\
\hline BLCA & $\begin{array}{l}9.93 e- \\
05\end{array}$ & $\begin{array}{l}6.17 \mathrm{e}- \\
04\end{array}$ & Tumor & 11.82 & 10.21 \\
\hline BRCA & $\begin{array}{l}5.98 \mathrm{e}- \\
05\end{array}$ & $\begin{array}{l}1.61 \mathrm{e}- \\
04\end{array}$ & Tumor & 10.98 & 10.68 \\
\hline COAD & $\begin{array}{l}7.53 e- \\
03\end{array}$ & $\begin{array}{l}1.78 \mathrm{e}- \\
02\end{array}$ & Tumor & 10.61 & 9.57 \\
\hline ESCA & $\begin{array}{l}7.78 \mathrm{e}- \\
03\end{array}$ & $\begin{array}{l}3.89 \mathrm{e}- \\
02\end{array}$ & Tumor & 10.55 & 10.14 \\
\hline HNSC & $\begin{array}{l}7.69 \mathrm{e}- \\
05\end{array}$ & $\begin{array}{l}2.63 e- \\
04\end{array}$ & Tumor & 11.35 & 10.96 \\
\hline $\mathrm{KICH}$ & $\begin{array}{l}1.06 e^{-} \\
03\end{array}$ & $\begin{array}{l}3.05 \mathrm{e}- \\
03\end{array}$ & Tumor & 10.99 & 10.49 \\
\hline KIRC & $\begin{array}{l}8.36 \mathrm{e}- \\
25\end{array}$ & $\begin{array}{l}2.30 \mathrm{e}- \\
23\end{array}$ & Tumor & 10.68 & 9.95 \\
\hline KIRP & $\begin{array}{l}1.54 \mathrm{e}- \\
05\end{array}$ & $\begin{array}{l}7.80 \mathrm{e}- \\
05\end{array}$ & Tumor & 10.60 & 10.13 \\
\hline LIHC & $\begin{array}{l}1.12 \mathrm{e}- \\
10\end{array}$ & $\begin{array}{l}2.40 \mathrm{e}- \\
09\end{array}$ & Tumor & 11.67 & 10.90 \\
\hline LUAD & $\begin{array}{l}1.52 \mathrm{e}- \\
04\end{array}$ & $\begin{array}{l}7.47 \mathrm{e}- \\
04\end{array}$ & Tumor & 11.68 & 11.20 \\
\hline LUSC & $\begin{array}{l}1.97 e- \\
05\end{array}$ & $\begin{array}{l}7.41 \mathrm{e}- \\
05\end{array}$ & Tumor & 11.54 & 11.06 \\
\hline PRAD & $\begin{array}{l}9.20 \mathrm{e}- \\
06\end{array}$ & $\begin{array}{l}4.29 \mathrm{e}- \\
05\end{array}$ & Tumor & 10.58 & 10.26 \\
\hline READ & $\begin{array}{l}8.54 \mathrm{e}- \\
03\end{array}$ & $\begin{array}{l}4.23 e- \\
02\end{array}$ & Tumor & 11.28 & 8.51 \\
\hline STAD & $\begin{array}{l}3.86 \mathrm{e}- \\
11\end{array}$ & $\begin{array}{l}2.92 \mathrm{e}- \\
09\end{array}$ & Tumor & 11.25 & 10.11 \\
\hline UCEC & $\begin{array}{l}9.47 e- \\
08\end{array}$ & $\begin{array}{l}7.54 \mathrm{e}- \\
07\end{array}$ & Tumor & 11.41 & 10.22 \\
\hline
\end{tabular}

BLCA: bladder urothelial carcinoma; BRCA: breast invasive carcinoma; COAD: colon adenocarcinoma; ESCA: esophageal carcinoma; HNSC: head and neck squamous cell carcinoma; KICH: kidney chromophobe; KIRC: kidney renal clear cell carcinoma; KIRP: kidney renal papillary cell carcinoma; LIHC: liver hepatocellular carcinoma; LUAD: Iung adenocarcinoma; LUSC: lung squamous cell carcinoma; PRAD: prostate adenocarcinoma; READ: rectal adenocarcinoma; STAD: stomach adenocarcinoma; UCEC: uterine corpus endometrial carcinoma

The expression of EMT marker protein was verified 24 hours after the transfection of negative control and miRNA151a-3p in the cell through the western blot. E-cadherin, a representative epithelial phenotype marker, was reduced in the miRNA151a-3p group. There was a significant increase in the mesenchymal phenotype 
marker vimentin and $\mathrm{N}$-cadherin moving inversely to $\mathrm{E}$-cadherin, and a noticeable increase in the expression of Twist1 associated with p53 metastasis (Fig. 6A, B) [25, 26]. For functional evaluation that induces the progression of cancer of miR-151a-3p, proliferation assay, Transwell migration, and invasion assay were implemented. A significant increase in proliferation was identified in the miRNA151a-3p 100nM group, depending on the concentration of miRNA151a-3p (Fig. 6C). Similarly, the cell movement and the increase in metastasis were identified depending on the concentration of miRNA151a-3p in cell migration, Invasion assay using transwell (Fig. 6D). The migrated and invasive cells were dyed and expressed in a graph after counting (Fig. 6E). So, evidence of the above data has demonstrated that miRNA151a-3p can induce EMTlike phenotype and increase cell proliferation.

In order to confirm the tumor progression ability of miRNA151a-3p in vivo, a nude mouse subcutaneous xenograft model experiment was conducted. HepG2 cells were injected $5 \times 10^{\wedge} 6$ subcutaneously in the flank of nude mice. Thereafter, miRNA-NC and miRNA-151a-3p / in vivo jet-PEI complexes were injected three times at 3 days intervals using the established in vivo jet-PEI transfection reagent to increase the expression of miRNA 151a-3p in cells [27]. In the in vivo experiment, the size of the tumor was significantly increased in miR-151a-3p compared to miRNA-NC, and the growth rate of the tumor was also faster (Fig. 7A-C). In addition, in western blot analysis in tumour tissue, miRNA-151a-3p significantly inhibited the expression of p53, P21, and Puma a/ $\beta$ (Fig. 7D, E). Comprehensive results of the experiment showed that overexpression of miR-151a-3p decreased the expression of the target protein in the tumour, and as a result, promoted tumour progression in the xenograft model.

\section{Discussion}

In-depth studies have shown that exosomes, which are widely present in various body fluids, contain many types of proteins, miRNAs, and various small molecules [3]. After secretion, exosomes are absorbed by adjacent or distant cells, and the miRNAs in them regulate processes such as promoting tumor growth, invasion, angiogenesis, and resistance to radiotherapy (RT). Therefore, miRNAs are promising as biomarkers in clinical targeted therapies in regulating cancer progression. [28]. Exosomes can be considered as modulators that target and bind underlying genetic molecules in the pathways regulating RT. $\mathrm{RT}$ is one of the important treatments for preventing tumor progression, and through various mechanisms, it affects target cancer tissue and surrounding cancer cells. Among them, p53 plays a key role in the suppression of cancer through RT [29],[30].

Our current study demonstrated a previously unknown interaction of p53 and HDAC5 in HCC by radiation exposure. Moreover, the expression of both proteins was confirmed in a time-dependent manner by radiation exposure. We confirmed that HDAC5 expression was increased by RT in p53-/-HepG2 cells, indicating that HDAC5 is an up-regulator of p53. These findings show that HDAC5 plays an important role in p53-mediated exosome release by radiation.

As previously studied, TSAP6 is known as a protein that regulates the secretion of exosomes [12]. TSAP6, exosome secretion modulating protein through $\mathrm{P} 53$, has increased expression in a radiation exposure environment. Changes of exosomes after radiation exposure were qualitatively and quantitatively analyzed

Page $17 / 30$ 
by Nanoparticle Tracking Analysis (NTA). The presence or absence of the p53 gene dramatically changed exosomes' secretion depending on the time of radiation exposure. In the previous figure, p53+/+ TSAP6 was most expressed after 12 hours of radiation exposure, and secretion of exosome was also most secreted after 12 hours of radiation exposure. On the contrary, the expression of TSAP6 by radiation exposure in the p53-/-cells did not change, and the secretion of exosomes after radiation exposure decreased. This suggests that TSAP6 is clearly involved in exosome secretion and that TSAP6 is regulated by changes in $\mathrm{p} 53$ resulting from radiation exposure. We photographed exosomes separated for exosome characterization using electron microscopy and analyzed western blot of exosome marker protein. Among the exosome marker proteins, Mammary Serine Protease Inhibitor (maspin) is a protein regulated by the p53 gene and functions to suppress cancer, and many studies are underway. Previous studies have confirmed that maspin inside the exosome is increased by radiation exposure in the lung cancer cell line [11]. We found that the expression of maspin increased significantly within the exosome secreted from p53+/+cells after radiation exposure. Besides, we confirmed that the expression of maspin did not increase in the p53-/-cells. Maspin is mounted on the exosome and released outside the cell, indicating that it functions to suppress tumor progression in surrounding cancer cells. Maspin is expected to suppress the cancer progression of the recipient cell.

The exosome is loaded with various substances (ex, protein, mRNA, miRNA., etc.) and plays an important role in communication between cells. Among them, miRNA is a potential biomarker, and many studies are underway [31] [32]. As a short RNA strand of the 18-22base pair, it binds to specific mRNAs with the same sequence and inhibits the gene's protein expression. After radiation exposure, changes in the miRNA composition balance inside the exosomes were observed. The change of miRNA inside exosome changed by radiation was thought to be the starting point for communication between cancer cells and changes in the cancer microenvironment.

Therefore, we generally performed a miRNA microarray to determine the miRNAs' overall expression in exosomes released in culture and exosomes released from irradiated cells. As a result of the analysis, 156 exosomal miRNAs were expressed out of a total of 2588 miRNAs. We classified exosomal-miRNAs for each cell function using GO term to determine which cellular function the changed exosomal-miRNAs are involved in after irradiation. As a result of classifying exosomal-miRNAs with a normalized expression (log2) of 1.5 or more, the number of exosomal-miRNAs involved in cell proliferation and cell migration decreased the number of exosomal-miRNAs involved in DNA repair and apoptosis increased. Among them, we conducted an analysis focusing on 33 miRNAs whose expression almost disappeared after irradiation. To find out the relationship between the disappeared 33 miRNAs and HCC, each miRNA expression was analyzed using the miRNA microarray dataset cohort in the cancer tissues of HCC patients. Among the 33 miRNAs that disappeared after irradiation, miR-151a-3p, miR-106b-5p, miR183-5p, and miR452-5p involved cell proliferation and invasion were significantly higher in expression in HCC cancer tissues.

So, we focused on miRNAs whose expression was reduced in exosomes after irradiation. As a result of analyzing using the GEO dataset (GSE74618) to find out how exosomal-miRNAs with decreased expression after irradiation were exhibited in cancer tissues of human liver cancer patients, 4 miRNA candidates with specifically increased expression were found. Four miRNA candidates showed a statistically significant 
increase in expression in liver cancer tissues. Subsequently, it was analyzed using the TCGA database (oncomiR) to analyze the association between miRNA candidates and liver cancer patients' survival rate. All miRNA candidates showed a direct correlation with the poor prognosis of liver cancer patients. These results are expected to contribute as a part of the mechanism for inhibiting cancer growth caused by radiation therapy.

MiRNA 151a-3p, one of the four miRNAs that had a significant relationship with the survival rate of liver cancer patients, was found to bind p53 with a high probability due to target protein analysis through the targetscan prediction tool. Subsequently, it was confirmed by qRT-PCR that the expression of miRNA151a$3 p$ was reduced in cells and exosomes due to radiation exposure. p53 is one of the key genes that maintain cell homeostasis by increasing expression by DNA damage such as radiation, leading to phenotypes such as cell cycle and cell death. As expected, miRNA151a-3p significantly reduced the expression of p53. Also, by inhibiting the expression of p53 downstream molecules P21 and Puma, which play an important role in the cell cycle and apoptosis in the p53 signaling pathway, it actually prevented cell cycle arrest and suppressed apoptosis after radiation exposure. Eventually, we confirmed that miRNA 151a-3p induces radiation resistance by inhibiting p53 signaling.

Subsequent experiments were conducted to determine the effect of miR151a-3p on cells. When miRNA151a-3p was transfected, Epithelial-mesenchymal transition (EMT)-like phenotype was verified by expression of related markers, increase of cell growth, migration, and invasion was discovered. The expression of Twist 1 , which indirectly inhibits cell cycle arrest and apoptosis functions regulated by p53, was increased [25, 33]. In our experimental results, in the miRNA151a-3p overexpression group, cell cycle arrest, and apoptosis were significantly reduced compared to the miRNA NC group. So, we proved that miRNA151a-3p induces anti-cell cycle arrest and anti-apoptosis effects on cells exposed to radiation.

\section{Conclusions}

Here, we confirmed the microRNA delivery mechanism through tumor-derived exosomes in RT. We have described the intercommunication occurring between irradiated and untreated cells via exosomal miRNAs that affect tumor proliferation. HDAC5 and p53 interacted by exposure to radiation, which increased exosome release and altered the composition of miRNAs within exosomes. In particular, the expression of exosomal miR151a-3p was markedly reduced as a result of the investigation. We confirmed that inhibition of exosomes miR151a-3p promotes apoptosis of non-irradiated cancer cells, thereby increasing radiotherapy sensitivity. Understanding the molecular mechanisms of exosome secretion and microRNA expression will help design novel therapeutics that improve sensitivity to RT in HCC. In conclusion, our present findings establish HDAC5 as a key component of the p53-mediated release of exosome in response to radiation treatment resulting in tumor apoptosis through exosomal miR-151a-3p resulting in improvement of RT.

\section{Abbreviations}


RT, radiotherapy; HDAC5, histone deacetylase 5; HCC, hepatocellular carcinoma; TSAP6, Tumor Suppressor Activation Pathway 6; miRNA, microRNA; EMT, Epithelial-mesenchymal transition.

\section{Declarations}

\section{Ethics approval and consent to participate}

Not applicable

\section{Consent for publication}

Not applicable

\section{Availability of data and material}

Not applicable

\section{Competing interests}

The authors declare that they have no competing interests.

\section{Funding}

This research was supported by National Research Foundation of Korea (NRF) grants (NRF-

2021R1A6A1A03040260, NRF-2020R1I1A2072110, NRF-2017M2A2A7A02020212). This work was also supported by grants (2018IP0600-1, 2019IP0848-1, 2020IL0006-1, 2021IP0048-1) from the Asan Institute for Life Sciences, Asan Medical Center, Seoul, Korea.

\section{Authors' contributions}

SML analyzed the data and developed the study. BHY., MHK, DHK, YHC, MC, JWL, TKK, JWR, KGK, YHS, CGP, and JKR collected and performed samples in the field. SWL, and CHH planed the study and wrote the manuscript with input from all of the authors. The authors read and approved the final manuscript. there are no conflicts where this is the case.

\section{Acknowledgements}

We thank the Confocal Microscope core facility and the Comparative Pathology Core at the ConveRgence mEDIcine research cenTer (CREDIT), Asan Medical Center for support and instrumentation. 


\section{References}

1. Mao L, Li X, Gong S, et al. Serum exosomes contain ECRG4 mRNA that suppresses tumor growth via inhibition of genes involved in inflammation, cell proliferation, and angiogenesis. Cancer Gene Ther. 2018;25(9-10):248-59.

2. Akers JC, Gonda D, Kim R, et al. Biogenesis of extracellular vesicles (EV): exosomes, microvesicles, retrovirus-like vesicles, and apoptotic bodies. J Neurooncol. 2013;113(1):1-11.

3. Sun Z, Shi K, Yang S, et al. Effect of exosomal miRNA on cancer biology and clinical applications. Mol Cancer. 2018;17(1):147.

4. Li X, Li C, Zhang L, et al. The significance of exosomes in the development and treatment of hepatocellular carcinoma. Mol Cancer. 2020;19(1):1.

5. Klemm F, Joyce JA. Microenvironmental regulation of therapeutic response in cancer. Trends Cell Biol. 2015;25(4):198-213.

6. Mashouri L, Yousefi H, Aref AR, et al. Exosomes: composition, biogenesis, and mechanisms in cancer metastasis and drug resistance. Mol Cancer. 2019;18(1):75.

7. Brooks $\mathrm{CL}, \mathrm{Gu}$ W. The impact of acetylation and deacetylation on the p53 pathway. Protein Cell. 2011;2(6):456-62.

8. Purvis JE, Karhohs KW, Mock C, et al. p53 dynamics control cell fate. Science. 2012;336(6087):14404.

9. Sen N, Kumari R, Singh MI, et al. HDAC5, a key component in temporal regulation of p53-mediated transactivation in response to genotoxic stress. Mol Cell. 2013;52(3):406-20.

10. Li T, Kon N, Jiang L, et al. Tumor suppression in the absence of p53-mediated cell-cycle arrest, apoptosis, and senescence. Cell. 2012;149(6):1269-83.

11. Yu X, Harris SL, Levine AJ. The regulation of exosome secretion: a novel function of the p53 protein. Cancer Res. 2006;66(9):4795-801.

12. Lespagnol A, Duflaut $D$, Beekman $C$, et al. Exosome secretion, including the DNA damage-induced p53dependent secretory pathway, is severely compromised in TSAP6/Steap3-null mice. Cell Death Differ. 2008;15(11):1723-33.

13. McKinsey TA, Olson EN. Toward transcriptional therapies for the failing heart: chemical screens to modulate genes. J Clin Invest. 2005;115(3):538-46.

14. Backs J, Olson EN. Control of cardiac growth by histone acetylation/deacetylation. Circ Res. 2006;98(1):15-24.

15. Zhang J, Shen L, Sun LQ. The regulation of radiosensitivity by p53 and its acetylation. Cancer Lett. 2015;363(2):108-18.

16. Citrin DE. Recent Developments in Radiotherapy. N Engl J Med. 2017;377(22):2200-1.

17. Chen CP. Role of Radiotherapy in the Treatment of Hepatocellular Carcinoma. J Clin Transl Hepatol. 2019;7(2):183-90. 
18. Ringuette Goulet C, Bernard G, Tremblay S, et al. Exosomes Induce Fibroblast Differentiation into Cancer-Associated Fibroblasts through TGFbeta Signaling. Mol Cancer Res. 2018;16(7):1196-204.

19. Zou Z, Gao C, Nagaich AK, et al. p53 regulates the expression of the tumor suppressor gene maspin. J Biol Chem. 2000;275(9):6051-4.

20. Martinez-Quetglas I, Pinyol R, Dauch D, et al. IGF2 Is Up-regulated by Epigenetic Mechanisms in Hepatocellular Carcinomas and Is an Actionable Oncogene Product in Experimental Models. Gastroenterology. 2016;151(6):1192-205.

21. Wong NW, Chen $Y$, Chen S, et al. OncomiR: an online resource for exploring pan-cancer microRNA dysregulation. Bioinformatics. 2018;34(4):713-5.

22. Bisio A, De Sanctis V, Del Vescovo V, et al. Identification of new p53 target microRNAs by bioinformatics and functional analysis. BMC Cancer. 2013;13:552.

23. Liu $H$, Cheng $Y, X u Y$, et al The inhibition of tumor protein $p 53$ by microRNA-151a-3p induced cell proliferation, migration and invasion in nasopharyngeal carcinoma. Biosci Rep 2019;39(10).

24. Kim DH, Park S, Kim H, et al. Tumor-derived exosomal miR-619-5p promotes tumor angiogenesis and metastasis through the inhibition of RCAN1.4. Cancer Lett. 2020;475:2-13.

25. Qin Q, Xu Y, He T, et al. Normal and disease-related biological functions of Twist1 and underlying molecular mechanisms. Cell Res. 2012;22(1):90-106.

26. Powell E, Piwnica-Worms D, Piwnica-Worms H. Contribution of p53 to metastasis. Cancer Discov. 2014;4(4):405-14.

27. Koo KH, Kwon H. MicroRNA miR-4779 suppresses tumor growth by inducing apoptosis and cell cycle arrest through direct targeting of PAK2 and CCND3. Cell Death Dis. 2018;9(2):77.

28. Chen R, Xu X, Tao Y, et al. Exosomes in hepatocellular carcinoma: a new horizon. Cell Commun Signal. 2019;17(1):1.

29. Torre LA, Bray F, Siegel RL, et al. Global cancer statistics, 2012. CA Cancer J Clin. 2015;65(2):87-108.

30. Butt Z, Parikh ND, Beaumont JL, et al. Development and validation of a symptom index for advanced hepatobiliary and pancreatic cancers: the National Comprehensive Cancer Network Functional Assessment of Cancer Therapy (NCCN-FACT) Hepatobiliary-Pancreatic Symptom Index (NFHSI). Cancer. 2012;118(23):5997-6004.

31. Zhao L, Liu W, Xiao J, et al. The role of exosomes and "exosomal shuttle microRNA" in tumorigenesis and drug resistance. Cancer Lett. 2015;356(2 Pt B):339-46.

32. Li S, Yao J, Xie M, et al. Exosomal miRNAs in hepatocellular carcinoma development and clinical responses. J Hematol Oncol. 2018;11(1):54.

33. Feng MY, Wang K, Song HT, et al. Metastasis-induction and apoptosis-protection by TWIST in gastric cancer cells. Clin Exp Metastasis. 2009;26(8):1013-23.

\section{Figures}




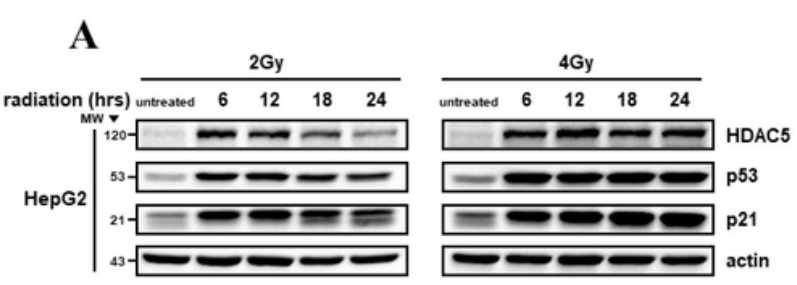

B

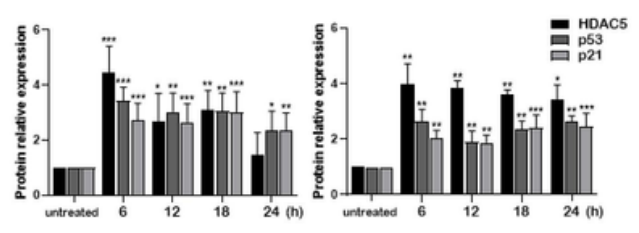

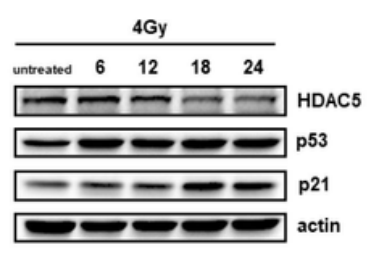

D

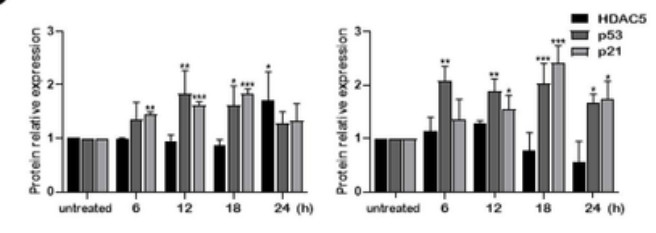

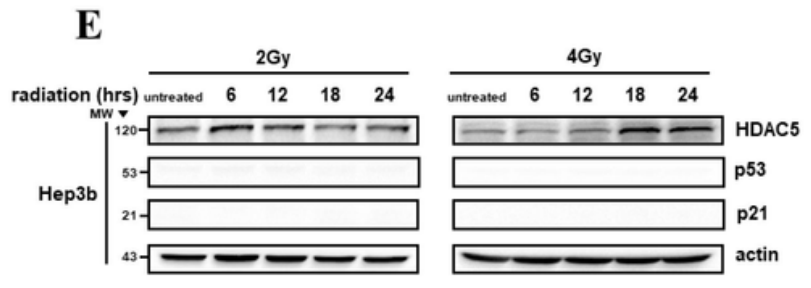

$\mathbf{F}$

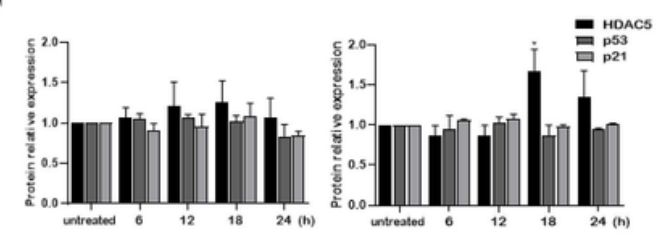

G

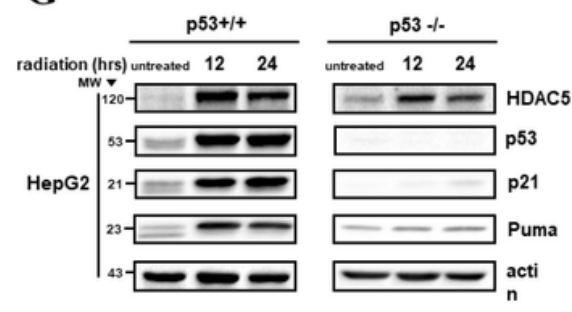

$\mathbf{H}$

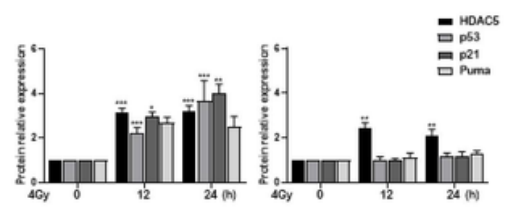

\section{Figure 1}

Modulation of protein p53 and HDAC5 expression by radiation exposure in hepatocellular carcinoma.

Radiation was exposed to 2Gy and 4Gy, and the protein expression of p53, P21, and HDAC5 were confirmed by Western blot analysis in a time-dependent manner up to 24 hours per six hours after exposure. (A-F). Using the CRISPER CAS9 system, a cell line with p53-/-in HepG2 was produced and the expression of HDAC5, p53, P21 and PUMA was confirmed through Western blot analysis after radiation exposure. The expression of each protein was quantified by b-actin. $(G, H)$ 
$\mathbf{A}$

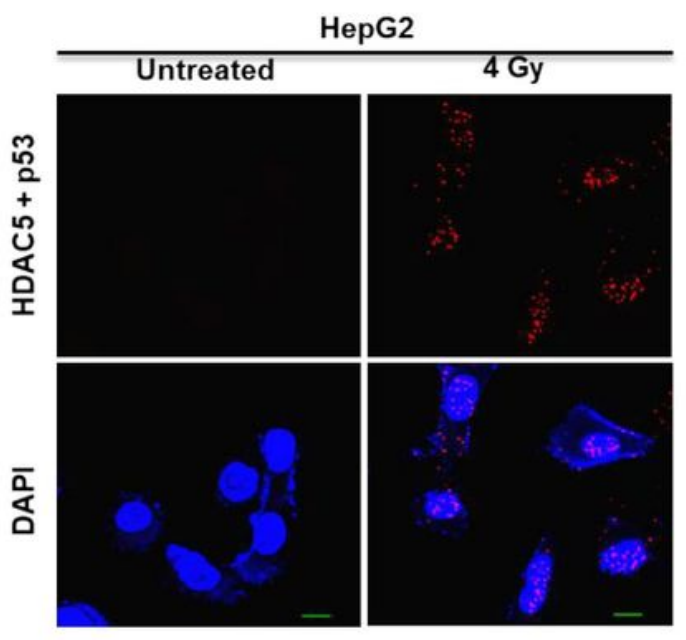

B
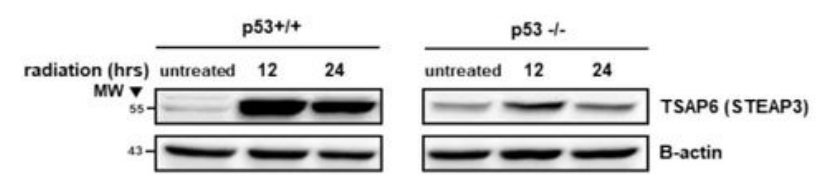

C

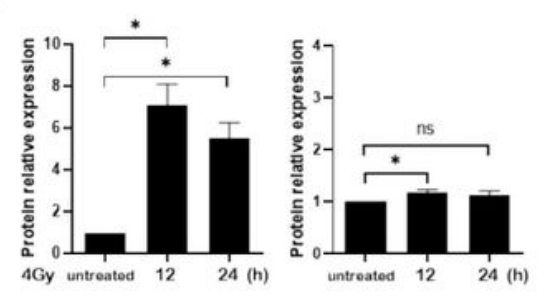

D

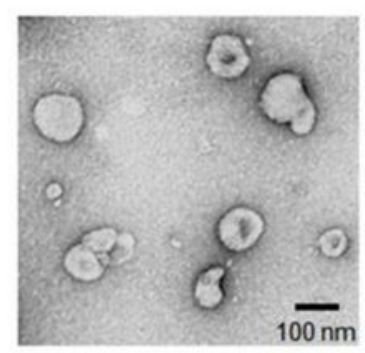

$\mathbf{E}$

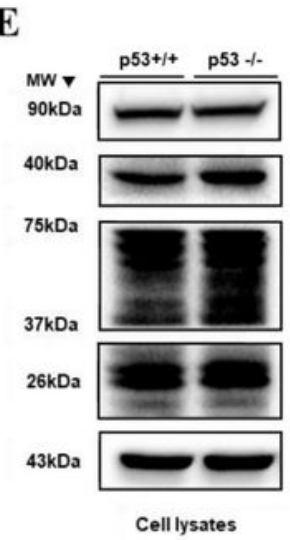

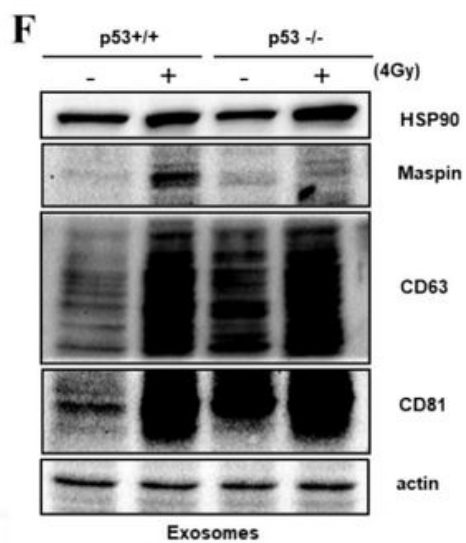

\section{Figure 2}

Interaction of HDAC5 and p53 by radiation exposure in HepG2 cells, and characterization of the exosome secretion pathway and the released exosomes that are altered by this union. The interaction between HDAC5 and p53 was confirmed after radiation exposure using Duolink proximity ligation assay in HepG2 (A). The expression of TSAP6 (STEAP3) by radiation exposure was confirmed by Western blot analysis. The expression of TSAP6 (STEAP3) was quantified by b-actin $(B, C)$. Electron microscopy was used to image resuspended exosome particles isolated from HepG2 (D). The expression of exosome marker protein 
HSP90, CD63, CD81 and Maspin with each sample 10ug after radiation exposure was verified with western blot analysis. (E,F).
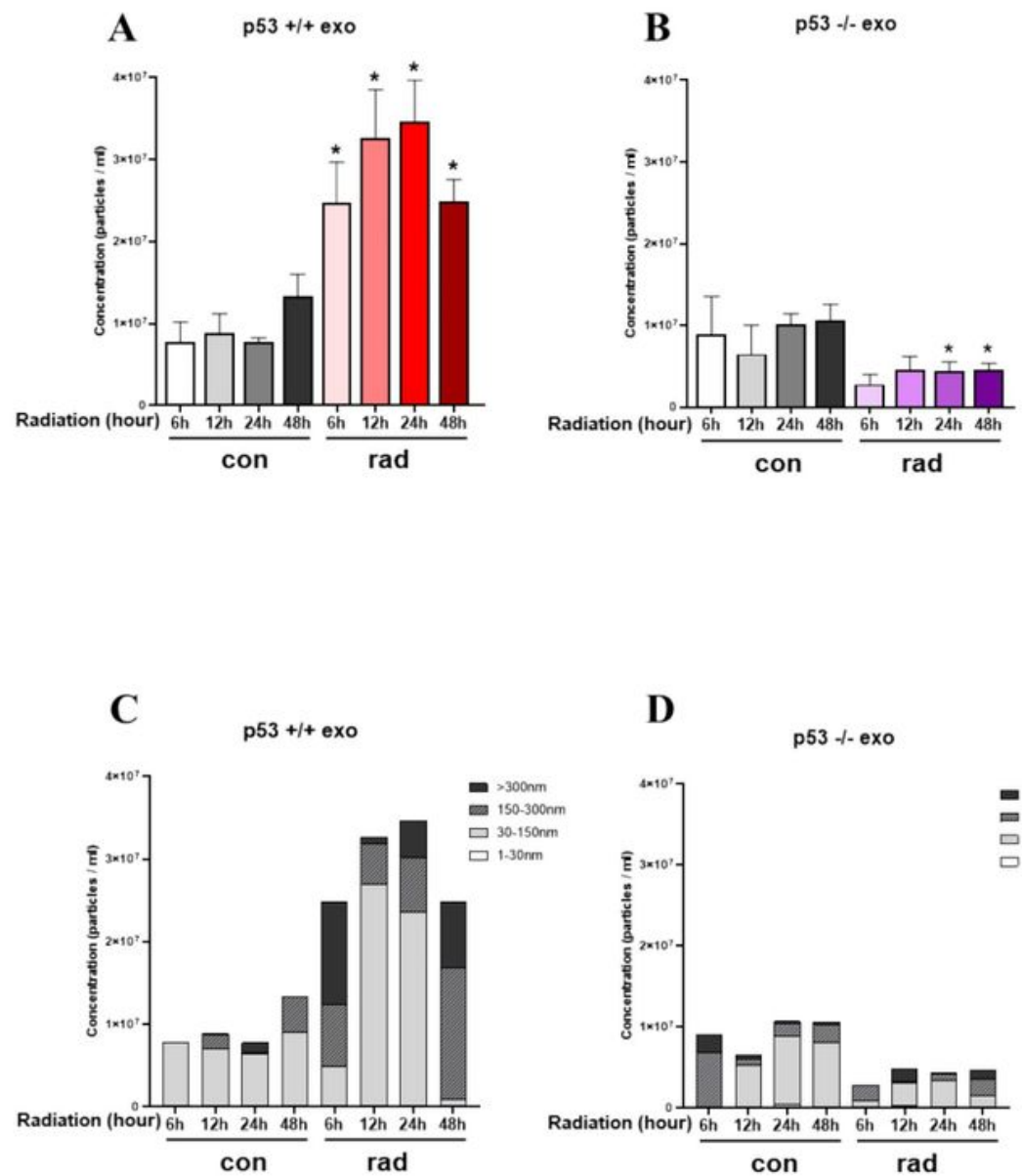

D

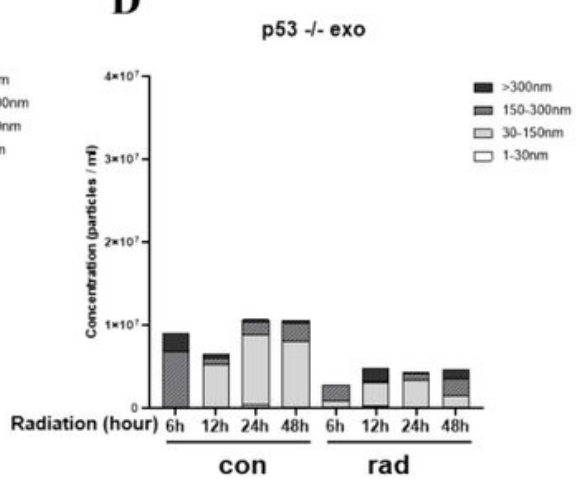

E

$\mathbf{F}$
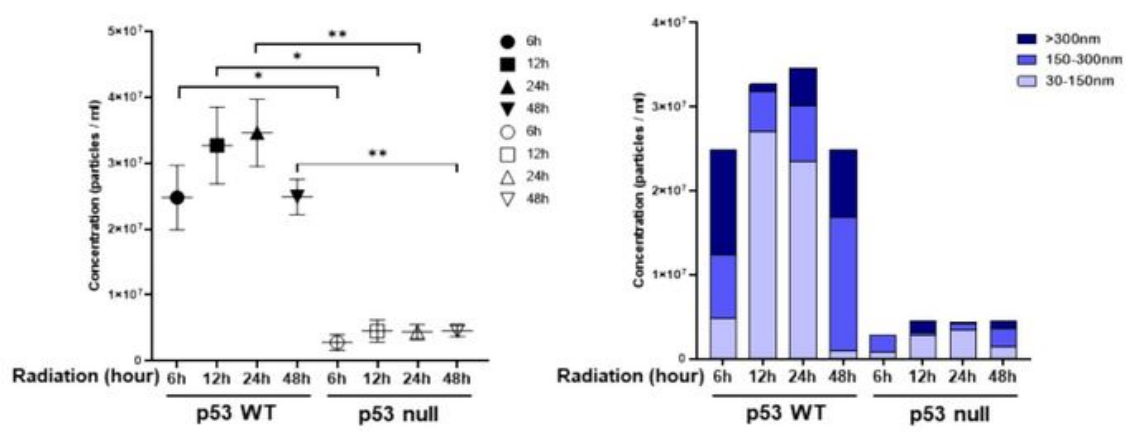

\section{Figure 3}

Time-dependent measurement and identification of the difference in exosome size and release amount determined by the p53 gene in liver cancer cells. Time-dependent measurements were made to determine whether the secretion of extracellular vesicles (EV) changes after radiation exposure using Nanosight's Nanoparticle Tracking Analysis (NTA) (A-B). Based on the analyzed nanosight data, three parts (30-150nm, 
150-300nm, $>300 \mathrm{~nm}$ ) were displayed by vesicle size (C,D). The secretion of EVs for the presence or absence of Tp53 genes after radiation exposure was analyzed qualitatively and quantitatively (E,F).

A
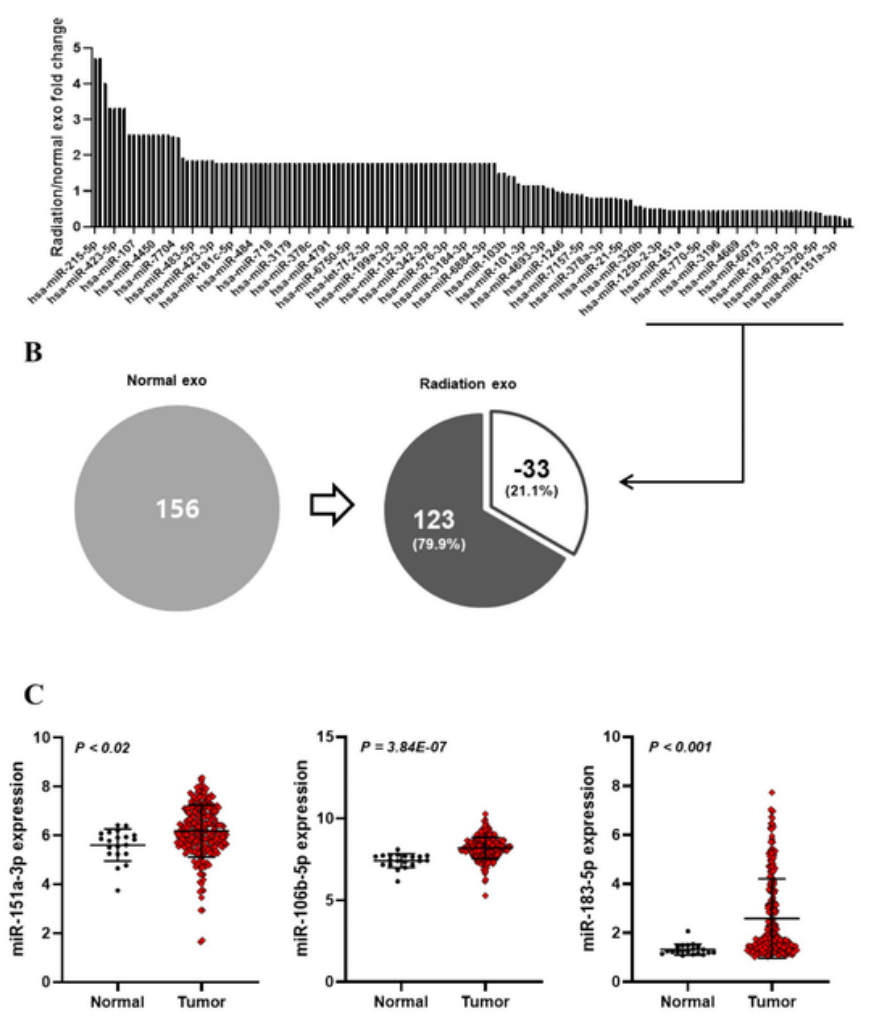

D TCGA(LIHC)
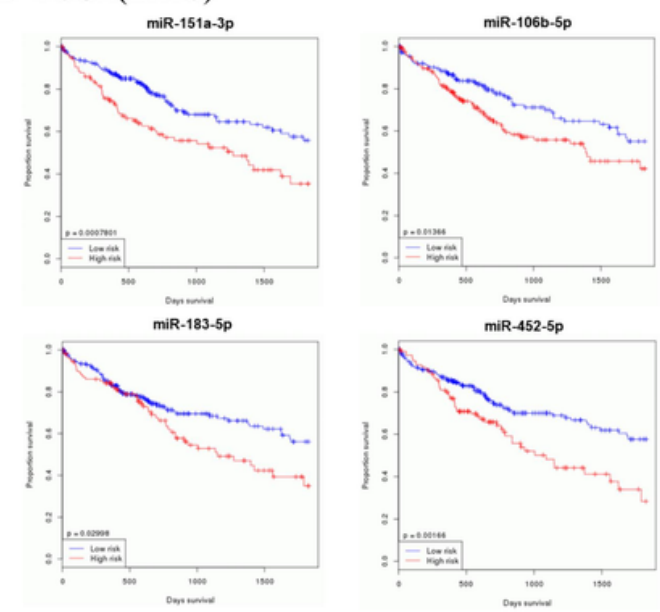

E

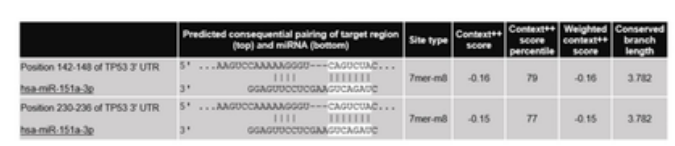

$\mathbf{F}$

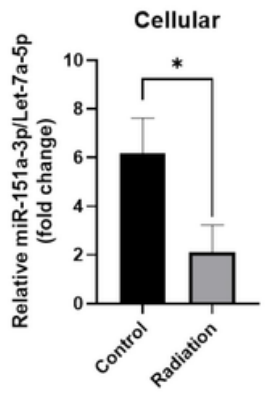

G

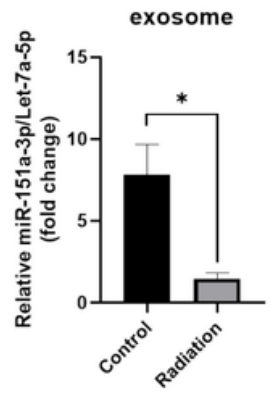

\section{Figure 4}

The expression analysis of exosomal miRNAs altered by irradiation in liver cancer cells and the clinical meaning exploration and identification of candidates using bioinformatics analysis. Before and after radiation exposure, exosomal miRNA was arranged into a fold change value and analyzed as a graph and ven diagram (A,B). Applying the GEO data set, the expression difference between normal and cancer of each candidate miRNA was analyzed. (C). To investigate the clinical significance of each candidate, it was analyzed using OncomiR Cancer miRNome Atlas and patient survival was expressed by Kaplan-Meier analysis (D). The target protein prediction analysis of the major candidate miR-151a-3p was performed by the Targetscan analysis tool (E). The modulation of a miR-151a-3p the radiation exposure was analyzed by qRT-PCR in the cell and the exosome. $(F, G)$. 
A

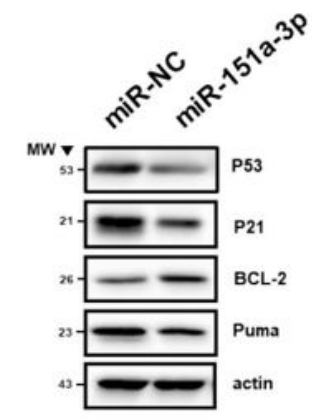

C
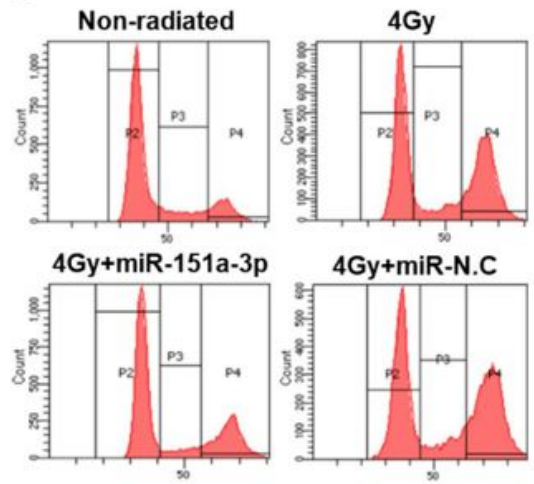

$\mathbf{E}$

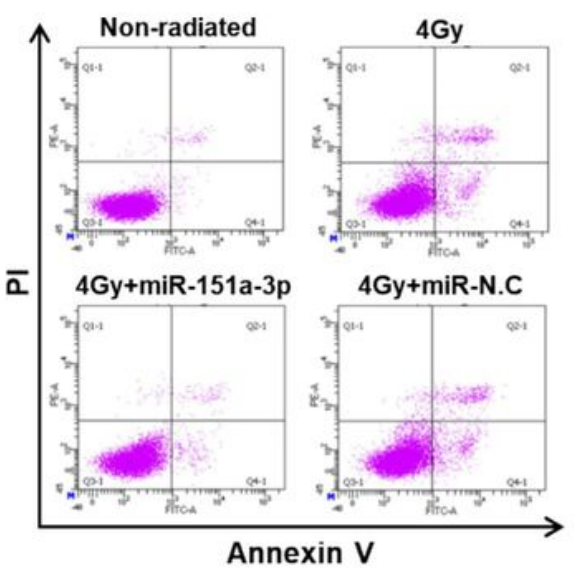

B

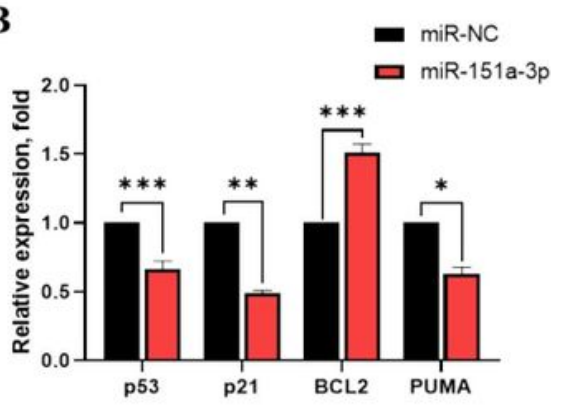

D

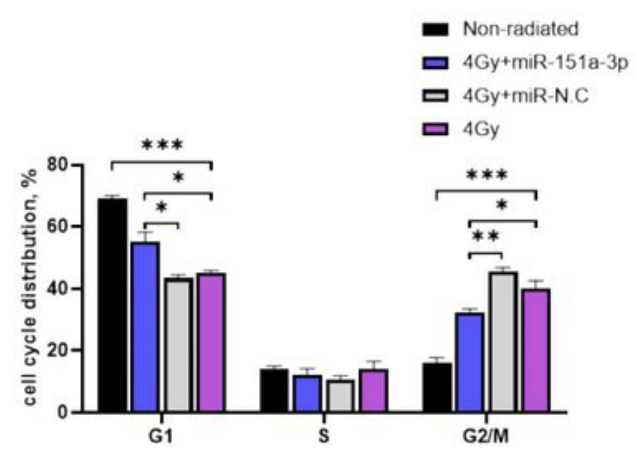

F

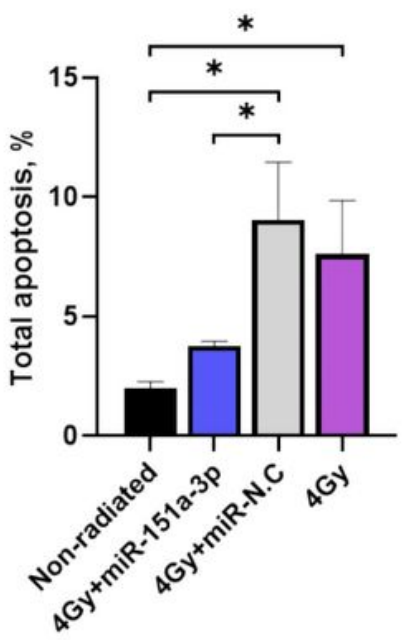

\section{Figure 5}

Cell dysfunction according to radiation exposure is regulated by miR151a-3p. Western blot analysis was performed to confirm that p53 and its sub-signals P21, PUMA, and BCL-2, which are closely related to cancer suppression, are modulated by miR151a-3p. $(A, B)$ Cell cycle arrest assay using FACS was performed by PI staining to confirm that cell cycle arrest induced by radiation exposure was modulated by miR151a3p. The Cell cycle was analyzed by dividing into G1, S, and G2 / M phases. (C,D) To confirm that cell 
apoptosis induced by radiation exposure is modulated by miR151a-3p, it was analyzed using FACS by Annexin V (FITC), PI staining. (E,F)

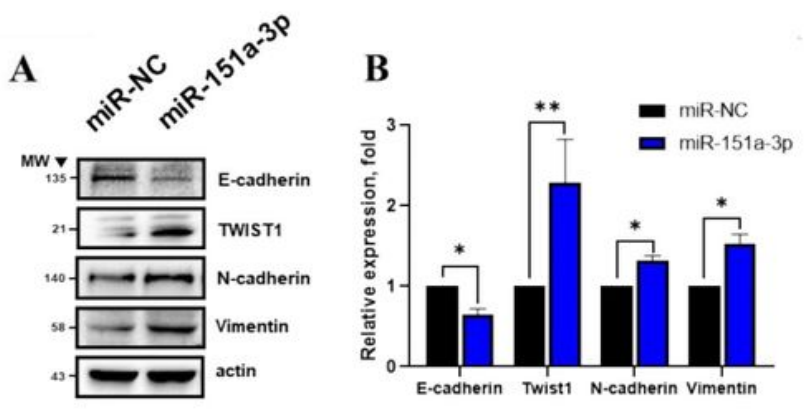

C

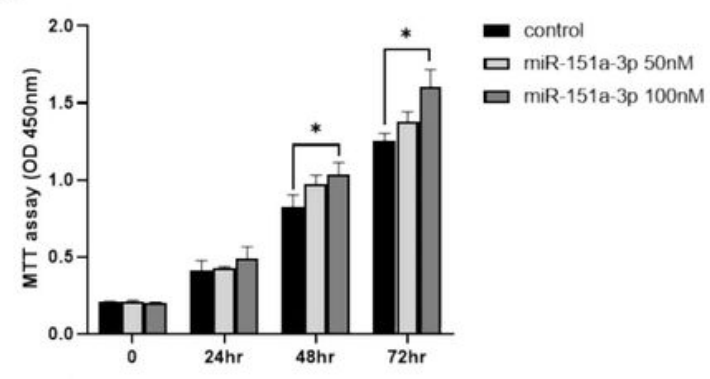

D

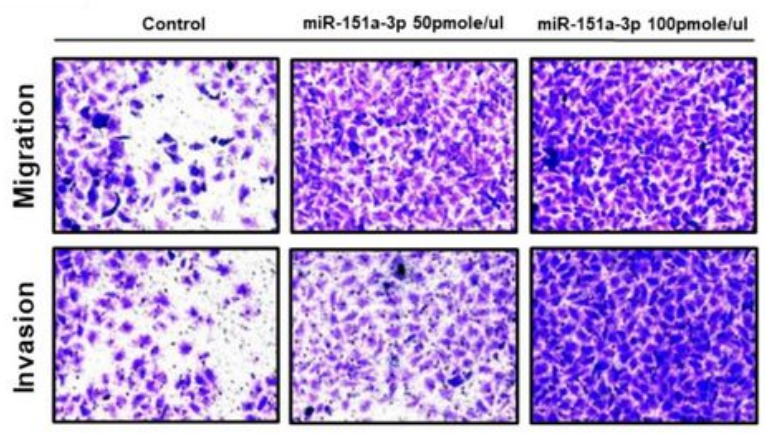

$\mathbf{E}$

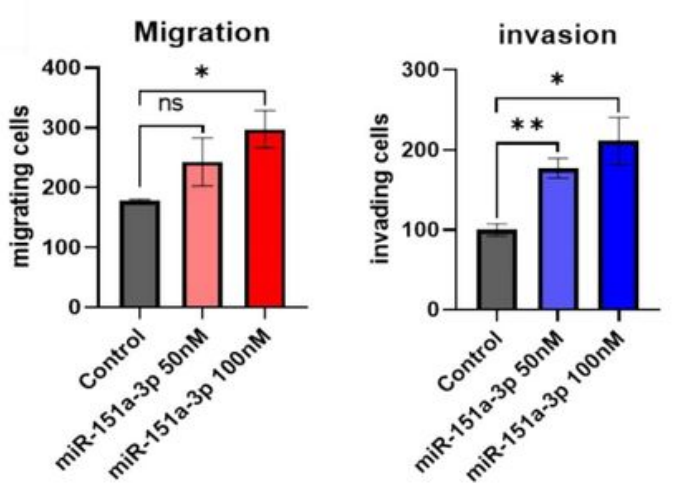

Figure 6

The ability of miRNA151a-3p to tumorigenesis. That the representative Epithelial-mesenchymal transition (EMT) marker of E-cadherin, Twist1, Vimentin, N-cadherin is regulated by the miR151a-3p was confirmed by Western blot analysis. $(A, B)$ The cell proliferation according to the concentration of the miR-151a-3p was 
analyzed by MTT assay. (C) Cell migration and invasion ability according to the concentration of miR-151a$3 p$ were analyzed using transwell assay. $(D, E)$
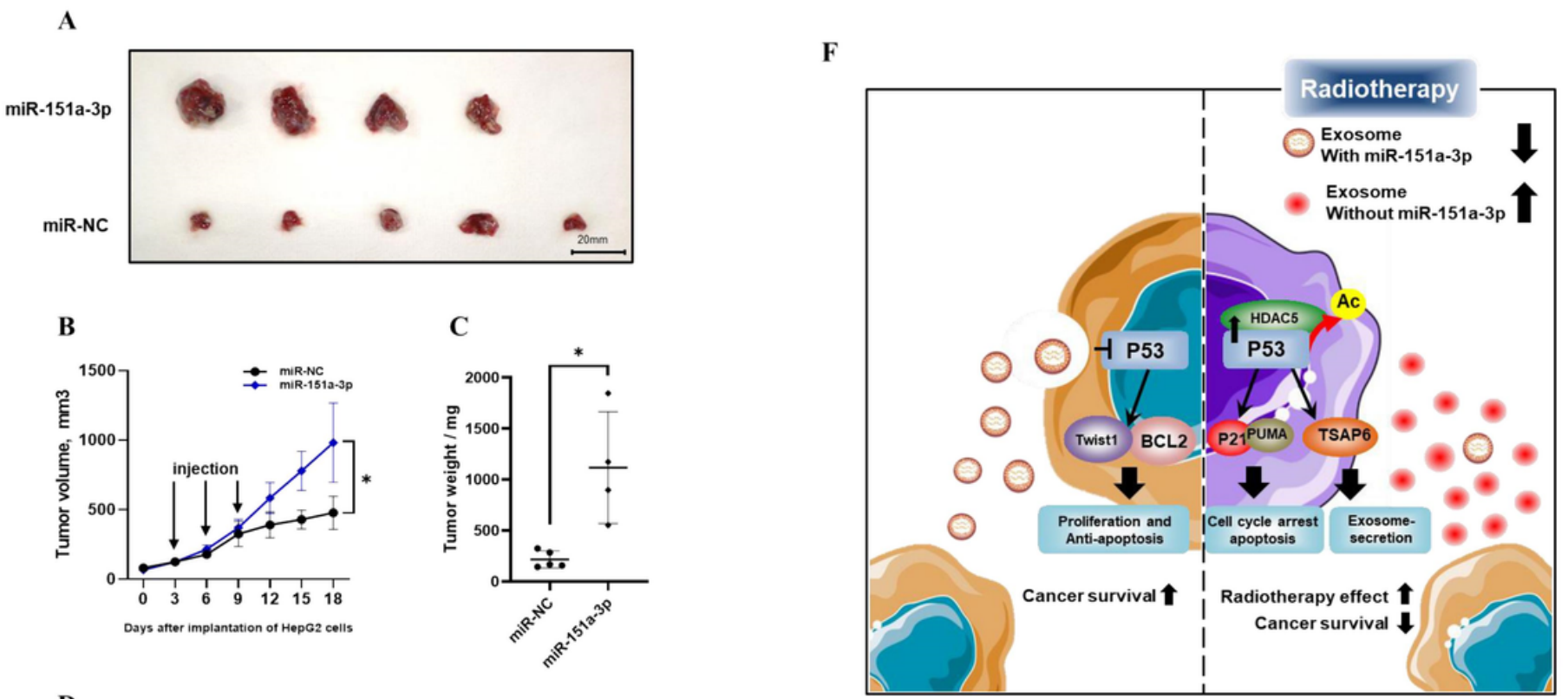

D

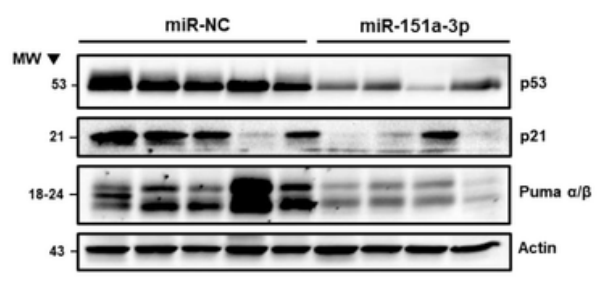

E

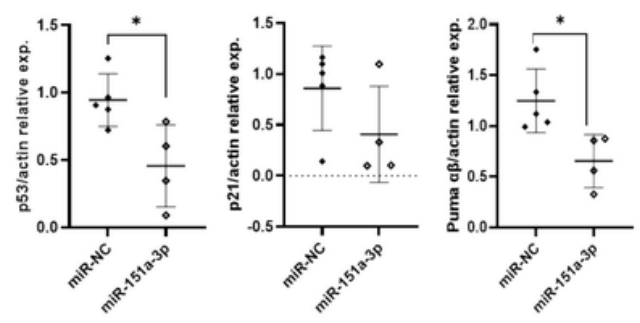

\section{Figure 7}

Increased tumor progression in a nude mouse subcutaneous tumor transplant model overexpressing miRNA 151a-3p Acceleration of tumor growth by overexpression of miRNA 151a-3p in a subcutaneous xenograft model. $(n=5)(A-C)$ Representative images at day 18 post-transplantation with a subcutaneous xenograft nude mouse model injected with miR 151a-3p/miR-NC. (A) After the mice were sacrificed on the 18th day, the tumor was separated and the size was compared, and the weight of the tumors of each group was measured and plotted. (B) In the subcutaneous xenograft model, miR-151a-3p, miR-NC, and invivo jetPEI complex were injected three times every three days from the third day after tumor transplantation, and the size of the tumors of each group was measured every three days. (C) The expression of the related target protein and downstream in tumour tissues were analyzed by Western blot. (D,E) A schematic image 
of Radiation-induced exosomal miRNA-151a-3p restrain hepatocellular carcinoma progression: By radiation, HDAC5 and p53 in HCC cells increase the secretion of exosomes as a result of the interaction. The exosomal miRNA-151a-3p inside the secreted exosome functions to infiltrate the surrounding recipient cells and promote the progression of cancer. $(\mathrm{F})$

\section{Supplementary Files}

This is a list of supplementary files associated with this preprint. Click to download.

- SupplementaryFigures.docx 